EXTENDED REPORT

\title{
A comparison of two primary care trials on tennis elbow: issues of external validity
}

\author{
N Smidt, M Lewis, E M Hay, D A W M Van der Windt, L M Bouter, P Croft
}

Ann Rheum Dis 2005;64:1406-1409. doi: 10.1136/ard.2004.029363

See end of article for authors' affiliations

Correspondence to:

Dr Nynke Smidt, Institute for Research in Extramural Medicine, VU University Medical Centre, Van der Boechorststraat 7, 1081 BT Amsterdam, Netherlands; n.smidt@vumc.nl

Accepted 21 March 2005 Published Online First 30 March 2005 geneity across two studies with respect to study population, Objective: To assess clinical heterogeneity across two studies with respect to study population,
interventions, and outcome measures, and to evaluate the influence of these sources of heterogeneity interventions, and outcome
on the results of the studies.

Methods: The individual patient data were used from two randomised controlled trials investigating the effectiveness of conservative treatments in patients with tennis elbow in primary care. Patients were allocated at random to treatment with steroid injection, wait and see policy, non-steroidal antiinflammatory drugs, placebo tablets, or physiotherapy. Outcome measures included severity of the main complaint, inconvenience of the elbow complaints, pain during the day, elbow disability, pain-free grip strength, and global improvement. All outcomes were assessed at 1, 6, and 12 months after randomisation.

Results: The two study populations were similar with respect to age, sex, comorbid neck/shoulder complaints, and baseline scores for the severity of pain. However, significant differences were observed for employment status, duration of elbow complaints, dominant side affected, previous history of elbow complaints, and use of analgesics. Local injections differed between the two studies with respect to volume, number, and steroid preparation. However, after 1, 6, and 12 months, the treatment effects of steroid injections were very similar between the study populations.

Conclusions: Despite large differences in study population at baseline, the responses to steroid injections were remarkably similar. Also the responses to other conservative interventions and the placebo treatment were very consistent, suggesting a uniform course of a tennis elbow and a lack of influence of clinical heterogeneity.
$\mathrm{L}$ ateral epicondylalgia (tennis elbow) is a common musculoskeletal problem. The incidence is estimated to be 4-7 per 1000 patients per year in general practice, ${ }^{1}$ and between $1 \%$ and $3 \%$ per year in the adult general population. ${ }^{2-5}$ Recently, Assendelft et al summarised the available evidence on the effectiveness of various treatment options for lateral epicondylalgia. ${ }^{6}$ They concluded that topical nonsteroidal anti-inflammatory drugs (topical NSAIDs) are effective for short term pain relief, and that oral NSAIDs are likely to be beneficial. Limited evidence was found for the short term effectiveness of steroid injections compared with placebo, local anaesthetic, elbow strapping, or physiotherapy. Although the randomised controlled trial (RCT) is the gold standard for evaluating the effectiveness of interventions, it is always open to question whether the results can be generalised to other populations.

We compared two large pragmatic randomised controlled trials in primary care investigating conservative treatments for lateral epicondylalgia that were remarkably similar in their outcome measures, interventions, and study populations. ${ }^{78}$ To investigate the generalisability of the results of these RCTs concerning the effectiveness of conservative treatments for lateral epicondylalgia, ${ }^{78}$ we determined the clinical heterogeneity across the two studies for study population, interventions, and outcome measures, and evaluated the influence of these sources of heterogeneity on the results of the studies.

\section{METHODS}

One RCT was carried out by Hay et al and compared the effectiveness of local injection of $20 \mathrm{mg}$ methylprednisolone plus lignocaine (lidocaine), naproxen $500 \mathrm{mg}$ twice daily for two weeks, and placebo tablets in 164 patients with a new episode of lateral epicondylalgia in North Staffordshire and South Cheshire, United Kingdom. ${ }^{7}$

The second RCT was carried out in Amsterdam and its vicinity in the Netherlands by Smidt et al. ${ }^{8}$ In this study, 185 patients with a new episode of lateral epicondylalgia were allocated either to a local injection of $10 \mathrm{mg}$ triamcinolone acetonide and lignocaine, physiotherapy, or a wait and see policy of ergonomic advice and pain medication. ${ }^{8}$

\section{Study population}

In both studies, consecutive patients who consulted their general practitioner (GP) for elbow pain were eligible for participation. The following inclusion criteria were applied in both studies: clinical diagnosis of lateral epicondylalgia; age between 18 and 70 years; and ability to fill in questionnaires and give informed consent.

Exclusion criteria in both studies included the following: contraindications to the treatments evaluated; previous surgery of the elbow; congenital or acquired deformities of the elbow; presence of signs and symptoms suggesting some other cause of lateral elbow pain (for example, cervical radiculopathy); and systemic musculoskeletal or neurological disorders. However, Hay et $a l^{7}$ excluded patients who had consulted their GP for their elbow pain during the preceding 12 months, whereas Smidt et $a l^{8}$ excluded patients who had been treated with physiotherapy or injection in the previous six months, patients with bilateral elbow complaints, and those with symptoms of less than six weeks' duration.

In both studies, patient characteristics and potential prognostic factors were recorded by a research nurse at the

Abbreviations: NSAID, non-steroidal anti-inflammatory drug; RCT, randomised controlled trial 
initial visit before randomisation. Demographic and clinical characteristics included age, sex, employment status, duration of the elbow complaints, presence of neck or shoulder pain, previous elbow complaints, and use of analgesics. The patients were also asked what they thought was the cause of their elbow complaint.

\section{Outcome measures}

Both studies assessed the following:

- the severity of the main complaint;

- inconvenience of the elbow complaints;

- pain during the day;

- elbow disability;

- pain-free grip strength at baseline and during follow up at 1,6 , and 12 months.

For three of these outcome measures, minor differences were found between the scales used in the two studies: severity of the main complaint, inconvenience of the elbow complaints, and pain during the day. Hay et $a l^{7}$ used a 10 point Likert scale whereas Smidt et al ${ }^{8}$ used an 11 point Likert scale. Also, different versions of the Pain-free Function Questionnaire ${ }^{9}$ were used to assess elbow disability: Hay et al $^{7}$ used the eight item tool with binary response options whereas Smidt et $a l^{8}$ used the 10 item tool including five point Likert options. To standardise scores across the two studies, all outcome measurements were transformed to 0 to 100 scales, where 100 indicates maximum severity.

The two studies used different instruments for the measurement of grip strength (hand held dynamometer by Hay et $a l^{7}$; Jamar hand dynamometer by Smidt et $\left.a l^{8}\right)$, and so for comparison purposes, the ratio of grip strength in the involved to the uninvolved arm was calculated and multiplied by 100 (a higher score indicates better grip strength).

General improvement was measured in both studies directly after the intervention period. However, Hay et $a l^{7}$ used a five point Likert scale (complete recovered, improved, no change, worse, much worse), whereas Smidt et $a l^{8}$ used a six point Likert scale (completely recovered, much improved, improved, no change, worse, much worse). For the computation of success rates, patients who rated themselves "completely recovered", "much improved", or "improved" were counted as successes.

\section{Treatments}

In the study of Hay et al, 53 patients were treated with a steroid injection, 53 received naproxen, and 58 received placebo tablets. ${ }^{7}$ In the study of Smidt et al, 64 patients were allocated to physiotherapy treatment, 62 received a steroid injection, and 59 were treated according to a wait and see policy. ${ }^{8}$

\section{Statistical analysis}

The data analysis was based on the individual patient data from both RCTs and was carried out using SPSS. ${ }^{10}$ Descriptive statistics were used to analyse differences in patient characteristics and outcome measures between the two study populations, and describe the course of symptoms. The Student $t$ test was used for ordinal and continuous outcomes and the $\chi^{2}$ test for dichotomous outcomes.

To compare treatment effects between the two trials, the following interventions were included: injection versus NSAID or placebo (NSAID and placebo had similar effect) ${ }^{7}$;

Table 1 Patient characteristics at baseline*

\begin{tabular}{|c|c|c|c|}
\hline & $\begin{array}{l}\text { Hay et al } \\
(n=164)\end{array}$ & $\begin{array}{l}\text { Smidt et al } \\
(n=185)\end{array}$ & $\begin{array}{l}\text { Difference between } \\
\text { studies† }(95 \% \mathrm{CI})\end{array}$ \\
\hline \multicolumn{4}{|l|}{ Sociodemographic characteristics } \\
\hline Age (years) (mean (SD)) & $48(9)$ & $48(10)$ & $0.2(-2$ to 2$)$ \\
\hline Female & $78(48 \%)$ & $93(50 \%)$ & $-2.7(-13$ to 8$)$ \\
\hline \multicolumn{4}{|l|}{ Employment status } \\
\hline manual worker & $73(45 \%)$ & $46(25 \%)$ & $19.6(10$ to 30$)$ \\
\hline non-manual worker & $51(31 \%)$ & $77(42 \%)$ & $-10.5(-21$ to -0.5$)$ \\
\hline unemployed & $40(24 \%)$ & $62(34 \%)$ & $-9.1(-19$ to 0.3$)$ \\
\hline \multicolumn{4}{|l|}{ Clinical characteristics } \\
\hline Duration of elbow complaints (weeks) (mean (SD)) & $12(15)$ & $18(27)$ & $-5.4(-10$ to -1$)$ \\
\hline One elbow affected onlyt & $149(91 \%)$ & $185(100 \%)$ & $-9.1(-)$ \\
\hline Dominant side affected & $100(63 \%)$ & $145(78 \%)$ & $-15.1(-25$ to -6$)$ \\
\hline \multicolumn{4}{|l|}{ Patients' attributed causes of elbow complaints: } \\
\hline unexpected movement & - & $4(2 \%)$ & - \\
\hline overuse, unusual activities & $24(15 \%)$ & $45(24 \%)$ & $-9.7(-18$ to -2$)$ \\
\hline overuse, usual activities & $33(20 \%)$ & $59(32 \%)$ & $-11.8(-21$ to -3$)$ \\
\hline accident & $17(10 \%)$ & $3(2 \%)$ & 8.7 (4 to 14$)$ \\
\hline sport injury & $12(7 \%)$ & $21(11 \%)$ & $-4.0(-10$ to 2$)$ \\
\hline other & - & $3(2 \%)$ & - \\
\hline cause unknown & $78(48 \%)$ & $50(27 \%)$ & 20.5 (11 to 31$)$ \\
\hline Concomitant neck or shoulder pain & $56(34 \%)$ & $58(31 \%)$ & $2.8(-7$ to 13$)$ \\
\hline Previous elbow complaints & $32(20 \%)$ & $58(31 \%)$ & $-11.8(-21$ to -3$)$ \\
\hline Analgesics in previous 48 hours & $118(72 \%)$ & $16(9 \%)$ & 63.3 (55 to 71$)$ \\
\hline \multicolumn{4}{|l|}{ Baseline measures of pain and function $\ddagger$} \\
\hline Severity of main complaint (mean (SD)) & $59.3(27.3)$ & $67.4(20.4)$ & $-8.1(-13$ to -3$)$ \\
\hline Inconvenience of elbow complaints (mean (SD)) & $39.4(24.1)$ & $67.5(23.9)$ & $-28.1(-33$ to -23$)$ \\
\hline Pain during the day (mean (SD)) & $56.3(24.2)$ & $54.3(21.1)$ & $2.0(-3$ to 7$)$ \\
\hline Pain-free grip strength (mean (SD)) & $65.3(23.4)$ & $33.9(21.6)$ & 31.4 (27 to 36$)$ \\
\hline Elbow disability (mean (SD)) & $78.6(22.3)$ & $48.2(16.9)$ & $30.4(26$ to 35$)$ \\
\hline \multicolumn{4}{|c|}{$\begin{array}{l}\text { *Values are } \mathrm{n}(\%) \text { unless specified. } \\
\text { †Difference is mean difference (in percentages of mean scores) between the two studies and } 95 \% \text { confidence } \\
\text { intervals }(\mathrm{CI}) \text {. } \\
\text { †Mean differences ( } 95 \% \text { confidence interval) after adjustment for sociodemographic and clinical characteristics } \\
\text { (linear regression analyses) were as follows: severity of main complaint }-16.3(-23.0 \text { to }-9.5) \text {; inconvenience of } \\
\text { elbow complaints }-36.5 \text { ( }-43.5 \text { to }-29.5) \text {; pain during the day }-6.0(-12.5 \text { to } 0.4) \text {; pain-free grip strength } \\
34.9 \text { (28.4 to } 41.3) \text {; elbow disability } 28.1 \text { (22.5 to } 33.7) \text {. }\end{array}$} \\
\hline
\end{tabular}


and injection versus wait and see policy. ${ }^{8}$ For this analysis, the physiotherapy group was excluded, as it was only a treatment option in one of the trials. The main outcome measures in the two studies were "pain during the day" and global assessment of change ("improvement" versus "no improvement") at a six week follow up.

Multiple regression (linear regression for pain during the day, and logistic regression for global assessment of change), including the treatment $\times$ study interaction was used to test the differences in treatment outcome across study populations. If the interaction was not statistically significant, suggesting that the treatment effect was consistent across the two study populations, the interaction term was dropped and differences in treatment outcome were studied in the combined dataset.

In addition, in order to estimate and compare the overall effectiveness of the interventions during the one year follow up, the area under the curve (AUC) for "pain during the day" was calculated.

\section{RESULTS}

\section{Study population}

In all, 182 patients had consulted the participating general practices $(n=23)$ in the UK and $164(90.1 \%)$ had finally been enrolled in the trial. Reasons for exclusion were: no informed consent $(n=12)$, exclusion criteria $(n=5)$, and inclusion criteria $(\mathrm{n}=1)$. In the study of Smidt et al, $185(71.4 \%)$ of 259 patients consulting participating GPs $(n=85)$ in the Netherlands were finally included in the trial. Reasons for exclusion were: no informed consent $(n=17)$, exclusion criteria $(n=52)$, and inclusion criteria $(n=5)$.

Table 1 shows the demographic and clinical characteristics and measurements for the two studies at baseline. The study populations were similar with respect to age, sex, and comorbid neck and shoulder complaints. Mean age of the patients in both studies was 48 years and almost $50 \%$ of the study population were female.

However, significant differences were observed for employment status, duration of elbow complaints, proportion of patients with the dominant side affected, previous history of elbow complaints, and use of analgesics. Also, the patients' attributed causes of their elbow complaints were less often identified in the study by Hay et al. ${ }^{7}$

With respect to sociodemographic and clinical characteristics, the mean severity of pain at baseline was similar in the two study populations, but self reported disability was greater among participants in the study by Hay et al. ${ }^{7}$ By contrast, severity of the main complaint, severity of the inconvenience of elbow complaints, and the pain-free grip strength were

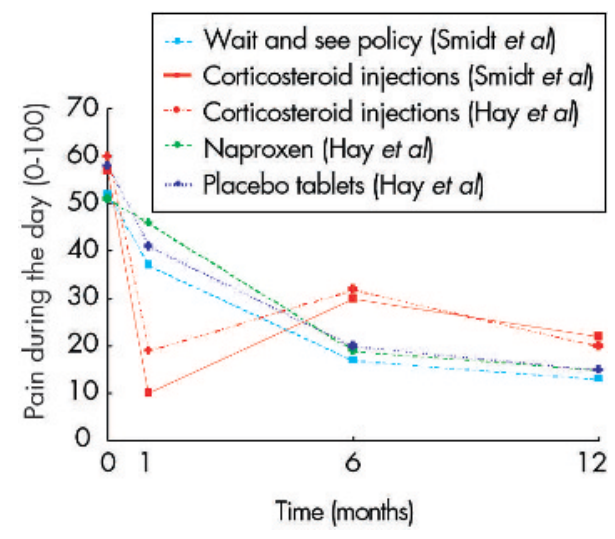

Figure 1 The severity of pain during the day at baseline and during follow up at 1, 6, and 12 months after randomisation for each intervention separately. significantly worse in the study by Smidt et al. ${ }^{8}$ There was little change in these baseline measures after controlling for differences in sociodemographic and clinical characteristics (table 1).

Figure 1 presents the course of "severity of pain during the day" for each intervention, except the physiotherapy treatment. After 12 months of follow up, a decrease in pain during the day was found for almost all patients (89\%), regardless of their treatment. The course of patients who received a corticosteroid injection $(\mathrm{n}=115)$ was remarkably similar in the two studies. After one month a large decrease in pain was found for patients treated with a corticosteroid injection. Many of these patients reported relapses or recurrences after six and 12 months of follow up. The course of pain during the day in patients who received NSAIDs, placebo tablets, and a wait and see policy was also quite similar (fig 1).

Overall at six weeks, there was improvement in 106 (65\%) of 164 patients in the UK and $158(85 \%)$ of 185 patients in the Netherlands. Also, for the pooled database, there was an improvement in 110 (96\%) of 115 patients given an injection and $101(59 \%)$ of 170 patients not given an injection. Regression analysis using the pooled databases indicated no significant interaction effect between treatment and study for both outcome measures (pain during the day and global assessment of change) ( $p>0.05)$. A greater proportion of the Dutch study population than of the UK population showed improvement by six weeks (adjusted odds ratio (OR) (95\% confidence interval $(\mathrm{CI}))=2.8(1.4$ to 5.4$))$, and less self reported pain (adjusted mean difference for pain during the day $=7.3(1.6$ to 13.0$))$.

The area under the curve (AUC) for the injection group $(\mathrm{n}=115)$ and no injection group (without physiotherapy) showed no significant differences between the studies, although the AUCs were lower (more pain relief) in Smidt's than in Hay's study (mean difference in AUC (95\% $\mathrm{CI})$ for the injection group $=101(-284$ to 487$)$; mean difference in AUC for the no-injection group $=115(-188$ to 419)].

\section{DISCUSSION}

The comparison of baseline patient characteristics between two randomised controlled trials recruiting patients with lateral epicondylalgia from primary care in different countries suggests that there are clinical differences between the study populations. However, the influence of the clinical heterogeneity between the studies on the effectiveness of conservative treatments for lateral epicondylalgia seems to be low, as the effectiveness of steroid injections and treatment with NSAID, placebo tablets, or a wait and see policy were similar in the two trials. There was a remarkable similarity between the course of pain in patients who received an injection in both studies. We do not know the reason for the high rate of relapses or recurrences in patients who received a steroid injection. For example, could it be that a steroid injection is actually harmful in the long term in patients with a non-inflammatory condition such as lateral epicondylalgia? Alternatively, do patients overtax their elbow too quickly after the initial dramatic reduction in pain following an injection?

The differences between the two studies in the duration of the elbow complaints and previous episodes of elbow complaints could be directly explained by disparities in the defined selection criteria of the two studies. Smidt et $a l^{8}$ excluded recent onset episodes (less than six weeks) and this was reflected in the longer average duration of elbow complaints in their study. In contrast, Hay et $\mathrm{al}^{7}$ excluded patients who had episodes of elbow complaints in the previous year, resulting in a lower prevalence of past elbow complaints in their study population. 
Furthermore, outcome measures used in both studies used different scales, which might explain the large differences in grip strength and elbow disability at baseline. The severity of pain during the day was the only outcome measure at baseline, which was comparable between the studies. To facilitate the interpretation and comparison of the results of various studies on lateral epicondylalgia, additional research is needed to standardise outcome measures. The incorporation of a standard set of outcome measures in all future RCTs on epicondylalgia would greatly enhance comparability of RCTs.

It is encouraging that recruiting patients from different countries and health care systems did not affect the important findings from these trials. Local corticosteroid injection was effective in the short term for treating lateral epicondylalgia but, crucially, does not improve long term outcome. The treatment effects in these two heterogeneous populations were similar for all interventions examined. These findings show that these trials have robust external validity and suggest that their results are generalisable to other primary care populations of patients with lateral epicondylalgia. $^{78}$

\section{ACKNOWLEDGEMENTS}

The study of Hay et al was funded by the Arthritis Research Campaign and the study of Smidt et al by the Netherlands Organisation for Scientific Research (NWO) and the Fund for Investigative Medicine of the Health Insurance Council. The collaboration project was funded by The Netherlands Organisation for Scientific Research
(NWO) and the North Staffordshire Primary Care Research Consortium.

\section{Authors' affiliations}

N Smidt, M Lewis, E M Hay, P Croft, Primary Care Science Research Centre, Keele University, Keele, Staffordshire, UK

N Smidt, D A W M Van der Windt, L M Bouter, Institute for Research in Extramural Medicine, VU University Medical Centre, Amsterdam, Netherlands

\section{REFERENCES}

1 Hamilton PG. The prevalence of humeral epicondylitis: a survey in general practice. J R Coll Gen Pract 1986:36:464-5.

2 Allander E. Prevalence, incidence and remission rates of some common rheumatic diseases or syndromes. Scand J Rheumatol 1974;3:145-53.

3 Kivi P. The aetiology and conservative treatment of humeral epicondylitis. Scand J Rehabil Med 1982:15:37-41.

4 Miedema HS. Rheumatology research (ROME): an initial report [In Dutch: Reuma-onderzoek meerdere echelons: basisrapport]. Leiden: Nederlands Instituut voor Praeventieve Gezondheidszorg TNO, 1994.

5 Verhaar JAN. Tennis elbow. Maastricht: University Press, 1992. [Thesis.]

6 Assendelft WJ, Green SE, Buchbinder R, Struiis PA, Smidt N. Tennis elbow (lateral epicondylitis). Clin Evid 2002;8:1290-300.

7 Hay EM, Paterson SM, Lewis M, Hosie G, Croff P. Pragmatic randomised controlled trial of local corticosteroid injection and naproxen for treatment of lateral epicondylitis of elbow in primary care. BMJ 1999:319:964-8.

8 Smidt N, Windt van der DAWM, Assendelft WJJ, Deville W, Korthals-de Bos I, Bouter LM. Corticosteroid injections, physiotherapy or a wait-and-see policy for lateral epicondylitis: a randomised controlled trial. Lancet 2002;359:657-62.

9 Stratford P, Levy DR, Levy K, Miseferi D. Extensor carpi radialis tendonitis: a validation of selected outcome measures. Physiother Can 1987:39:250-5.

10 SPSS for Windows, release 11.0. Chicago: SPSS, 2001. 